\title{
New information throws light on how Yurii Gagarin died
}

\section{London}

LAST week, Pravda published what appears to be the final verdict on the death of Yurii Gagarin, the first man in space. Gagarin and his co-pilot, Vladimir Seregin, died in an air crash in March 1968. The findings of the official inquiry were not, however, published at the time, and a partial account in Pravda last year concentrated on Gagarin's state of health before and during his last flight, and his heroic final efforts to wrestle the aircraft out of a near-vertical dive, leaving open the question of what had caused the dive.

The original silence had inevitably generated many rumours ranging from Gagarin being drunk at the controls to his having fallen from political favour and eliminated in a staged "accident". Last year's Pravda article, co-signed by a leading astronautics expert, Professor Sergei Belotserkovskii, and cosmonaut Aleksei Leonov, effectively dispelled these rumours. But the lack of a clear-cut expla- nation for the accident generated the rumours that there had been some kind of cover-up. During the past ten months, Pravda received many letters asking for more details. Last week, therefore, Belotserkovskii provided a further explanation

According to this new reconstruction, Gagarin and Seregin were in a Mig-15 jet coming in to land from an altitude of 4,200 $\mathrm{m}$ and were between two layers of cloud, with no visibility of the horizon. The flight controller had informed them that the height of the lower layer was $900 \mathrm{~m}$; it was in fact $400-500 \mathrm{~m}$. (The controller's figure was correct, but for the vicinity of the airfield, not for the flight zone.) As they were making their approach, a Mig-21 jet, far larger than the Mig-15, passed within some $500 \mathrm{~m}$ of Gagarin's aircraft. The Mig-21 pilot apparently never saw the Mig-15 or knew that it was nearby. The smaller aircraft, however, was hit by the jet-wash, and

\section{US telescope plans postponed until accuracy demands are met}

\section{Austin, Texas}

FACED with uncertain finances and some technical doubts, the National Optical Astronomy Observatory (NOAO) will not pursue, for the time being, plans to build the National New Technology Telescope (NNTT), a giant four-in-one interferometric array of mirrors. NOAO, which operates the Kitt Peak Observatory in Arizona and the Cerro Tololo Observatory in Chile, hopes instead to submit a proposal before the end of this year to build two 8-m telescopes, one on Mauna Kea, Hawaii, and the other somewhere in the Southern Hemisphere.

One participant in the decision said that it was a consequence of "realistic thinking" about the likelihood that the National Science Foundation (NSF) would provide funds for NNTT before it had been demonstrated that large mirrors of the required accuracy could be made.

Speaking here at this month's American Astronomical Society meeting, Sidney Wolff, director of NOAO, also had a wider caution for astronomers. After years of essentially level funding from NSF, most programmes had been trimmed to the bone, and the only alternative to a general "slide into mediocrity" might be to close down some facilities. No such decision has yet been made, but Wolff made it clear that she saw the habitual defence by all astronomers of all NOAO activities as a questionable strategy for the near future.

NOAO is expected to play a double role, providing astronomical facilities for general use but also leading the development of new techniques. Some observers at the meeting seemed to think that the

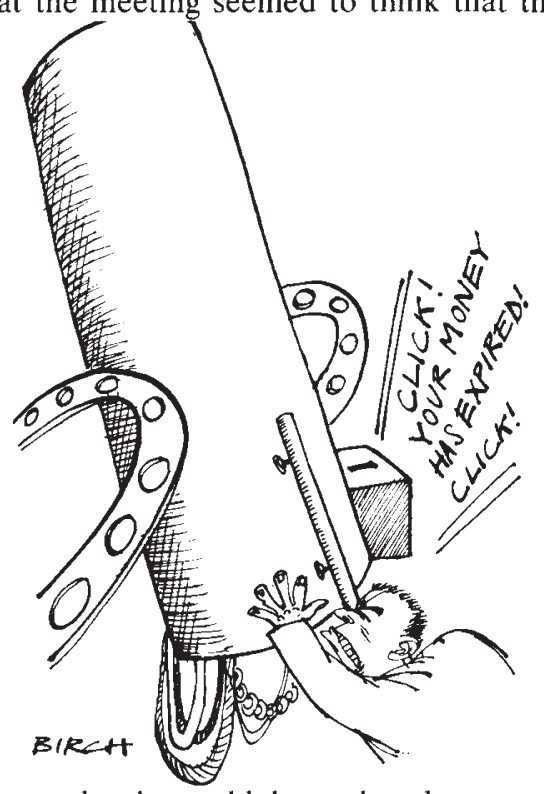

second role could be reduced or even abandoned, but Wolff was clearly unprepared to allow superiority to fall into the hands of the European Space Agency, which intends to go ahead with its own version of NNTT (see Nature 330, 593; 1987). began to dive. On the basis of the erroneous information, Gagarin and Seregin, who were still in the clouds, believed they had enough height to pull out of the dive. They were wrong — by a mere $250 \mathrm{~m}$, or 2 seconds.

This new scenario, Belotserkovskii stressed, has been established during the past ten months, using meteorological records, voice tapes computer simulations, and "personal archive materials". (Black box flight recorders, he explained, were not installed in Mig-15s at that time - a surprising revelation.) The fact that so much new information could be established almost 20 years after the accident, he explained, was not because of an earlier cover-up but to the fact that although the original commission of inquiry carried out "careful and profound investigations in all directions", the commission "did not carry its work through to the end". the two subcommissions into which it was divided did not coordinate their work properly, he said. Most important, there was no "group of competent experts" that could have studied and appraised all the data presented by the working groups, and drawn general conclusions from them. This, if glasnost continues, may evoke a further deluge of questions: why not? Vera Rich

\section{India modernizes crematoria \\ New Delhi}

WITH the Hindu custom of burning dead bodies in funeral pyres threatened by rising fuelwood prices due to rapid depletion of forests, the Indian government is seriously thinking of modernizing its crematoria.

Apart from cooking, fuelwood in India is used chiefly for cremation. A massive programme is already under way to introduce smokeless, fuel-efficient "chulas" (wood-stoves) in villages. And now the government has launched a scheme to install new designs of wood-burning crematoria twice as efficient as the existing ones.

Some 1,500 such specially designed crematoria have been installed in the state of Gujarat during the past five years under a project supported by the World Bank. They have shown a $\mathbf{5 0}$ per cent saving in firewood and a halving of the previous cremation time of three hours. The Energy Advisory Board recommends replacing all existing crematoria by the end of 1990 .

In Delhi alone, some $\mathbf{5 0 , 0 0 0}$ bodies are burned each year in 12 crematoria with 260 platforms, one cremation consuming a six months supply of cooking fuel for a family. The high cost of wood means the poor are forced to throw the dead bodies into rivers after partial burning. Along the banks of the Ganges, where floating bodies are common, the government is setting up 29 electric crematoria, eliminating the need for wood. K.S. Jayaraman 